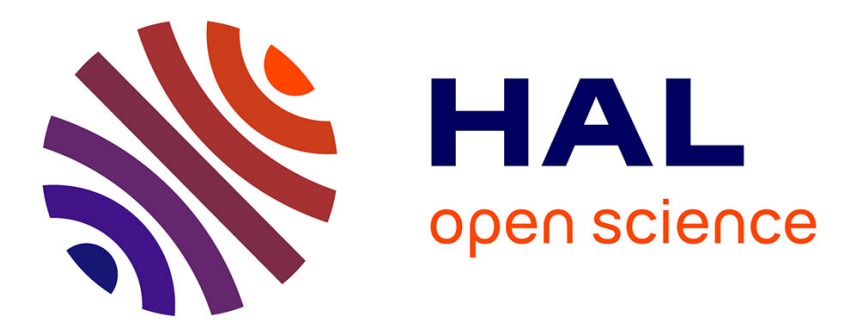

\title{
A Generic Framework for Modeling MAC Protocols in Wireless Sensor Networks
}

Fayçal Ait Aoudia, Matthieu Gautier, Michele Magno, Olivier Berder, Luca Benini

\section{- To cite this version:}

Fayçal Ait Aoudia, Matthieu Gautier, Michele Magno, Olivier Berder, Luca Benini. A Generic Framework for Modeling MAC Protocols in Wireless Sensor Networks. IEEE/ACM Transactions on Networking, 2017, 25 (3), pp.1 - 12. 10.1109/TNET.2016.2631642 . hal-01416008

\section{HAL Id: hal-01416008 \\ https://hal.inria.fr/hal-01416008}

Submitted on 13 Dec 2016

HAL is a multi-disciplinary open access archive for the deposit and dissemination of scientific research documents, whether they are published or not. The documents may come from teaching and research institutions in France or abroad, or from public or private research centers.
L'archive ouverte pluridisciplinaire HAL, est destinée au dépôt et à la diffusion de documents scientifiques de niveau recherche, publiés ou non, émanant des établissements d'enseignement et de recherche français ou étrangers, des laboratoires publics ou privés. 


\title{
A Generic Framework for Modeling MAC Protocols in Wireless Sensor Networks
}

\author{
Fayçal Ait Aoudia*, Matthieu Gautier*, Michele Magno ${ }^{\dagger}$, Olivier Berder*, Luca Benini ${ }^{\dagger}$ \\ *University of Rennes 1 , IRISA \\ ${ }^{\dagger}$ ETH Zürich
}

\begin{abstract}
Wireless sensor networks are employed in many applications such as health care, environmental sensing, and industrial monitoring. An important research issue is the design of efficient Medium Access Control (MAC) protocols, which have an essential role for the reliability, the latency, the throughput and the energy efficiency of communication, especially as communication is typically one of the most energy consuming tasks. Therefore, analytical models providing a clear understanding of the fundamental limitations of the different MAC schemes, as well as convenient way to investigate their performance and optimize their parameters, are required. In this work, we propose a generic framework for modeling MAC protocols, which focuses on energy consumption, latency and reliability. The framework is based on absorbing Markov chains, and can be used to compare different schemes and evaluate new approaches. The different steps required to model a specific MAC using the proposed framework are illustrated through a study case. Moreover, to exemplify how the proposed framework can be used to evaluate new MAC paradigms, evaluation of the novel pure-asynchronous approach, enabled by emerging ultra-low power wake-up receivers, is done using the proposed framework. Experimental measurements on real hardware were performed to set framework parameters with accurate energy consumption and latency values, to validate the framework, and to support our results.
\end{abstract}

\section{INTRODUCTION}

Wireless Sensor Networks (WSNs) have enabled a large number of applications ranging from body area health monitoring to wide-range environmental surveillance. The task of a WSN is to measure one or more environmental variables through sensors, to eventually pre-process this information, and to transmit the so obtained data to a sink, i.e. a device responsible of gathering the data generated by the sensor nodes. Typical WSNs usually have limited resources such as memory, computation capability and available energy. A central task in WSNs is wireless communication, and it has been shown in various design cases that communication is the most power hungry task [1].

Great efforts are made to design network protocols that fulfill the requirements of WSNs, especially regarding the Medium Access Control (MAC) layer [2]. The aim of the MAC layer is to provide mechanisms to allow several wireless nodes to share the wireless channel medium and access the network. The MAC layer fundamental tasks are to avoid collisions and to provide fair medium allocation among the nodes. Moreover, as the MAC layer controls the transceiver, it plays an important role in the energy efficiency of communication. Because WSNs have many different applications with different requirements, and as the choice of an appropriate protocol strongly depends on the application requirements, the amount of proposed MAC protocols is large and multiple MAC protocols categories were defined over the last decade [2]. As a consequence, it is difficult to choose and tune the most appropriate MAC protocol given a specific application context or to compare new MAC schemes to state-of-the-art, especially because of the lack of generic analytical models. Indeed, analytical models are required to investigate the performance of different schemes, to characterize their fundamental limitations and to optimize their parameters.

In this paper, a generic framework for modeling MAC protocols is presented. The proposed framework is based on Absorbing Markov Chains (AMCs) [3] and focuses on energy consumption, latency and reliability. Markov Chains (MCs) have already proved to be useful for modeling communications protocols, especially to study specific MAC protocols [4] and cross-layer designs [5]. The purpose of this generic framework is to permit the modeling of a wide range of MAC layer schemes to explore their parameter space and to compare them. To illustrate how to apply the proposed framework to model a specific MAC protocol, the state-of-the-art PWMAC [6] protocol, which is an improvement of RI-MAC [7], is modeled using the proposed framework, and the different modeling steps are explained. PW-MAC focuses on low energy consumption for both the receiver and the sender. Moreover, experimental measurements using real hardware were performed to set the model parameters using realistic and accurate energy consumption and latencies values and to validate the framework.

The generic framework proposed in this work can also be used to evaluate new paradigms. To exemplify this, we use the proposed framework to evaluate the pure-asynchronous approach enabled by emerging Ultra Low Power (ULP) Wakeup Receivers (WuRx). ULP WuRx constitute an emerging technology that allows continuous channel monitoring while consuming orders of magnitude less power than traditional transceivers [8]. These devices wake up the node microcontroller (MCU) or other sleeping subsystems using interrupts when a specific signal, called Wake-up Command (WuC), is detected. ULP WuRx can significantly increase the energy efficiency of communications and reduce the latency [9]-[13]. The framework proposed in this work is used to evaluate the benefits enabled by these new schemes, by modeling two simple MAC protocols that leverage ULP WuRx: TI-WuR and RI-WuR, both from [9]. These schemes are compared to PWMAC, X-MAC [14], a popular transmitter-initiated protocol, 
and to the IEEE 802.15.4 standard in its beaconless mode, which is based on the well-known Carrier Sense Multiple Access with Collision Avoidance (CSMA/CA) scheme. Experimental measurements were realized to support our results.

The principal contributions of this work are:

- A new generic framework for modeling MAC protocols based on AMCs.

- A detailed modeling of the PW-MAC protocol to illustrate the different steps required to model a MAC protocol using the proposed framework. The model parameters were set using experimental measurements to get realistic and accurate energy consumption and latencies values.

- Analytical and experimental evaluation of two emerging pure-asynchronous approaches by comparing them to three traditional MAC schemes.

- Experimental measurements realized to validate the accuracy of the proposed framework.

The rest of this paper is organized as follows. Section II exposes the related work. In Section III, the generic framework is presented. Section IV exposes the modeling of PW-MAC using the generic framework and the experimental validation of the model. In Section $\mathrm{V}$, an analytical and experimental comparison between two MAC schemes leveraging ULP WuRx (TI-WuR and RI-WuR) and three traditional MAC schemes (PW-MAC, X-MAC, and CSMA/CA) is presented. Finally, Section VI concludes this paper.

\section{RELATED WORK}

The most widespread communication scheme for WSNs is the standard IEEE 802.15.4, which defines both a physical layer and a MAC protocol. Consequently, many dedicated models were proposed for characterizing and evaluating this scheme [15]-[19]. In [15], the authors evaluated the energy efficiency of the IEEE 802.15.4 MAC layer in dense networks, and concluded that this standard can be used to support communication in dense data-gathering networks. Ramachandran et al. [16] evaluated the throughput and energy consumption of the contention access period of the IEEE 802.15.4 MAC layer by modeling it as a non-persistent CSMA with backoff, and showed that shutting down the radio between transmissions improves the energy efficiency of the MAC layer in some applications. In [17], the IEEE 802.15.4 MAC protocol was modeled using MCs, assuming perfect channel conditions, to capture the performance of the scheme in terms of throughput and energy consumption, and in the case of saturated and unsaturated networks. Similarly, Park et al. [18] modeled the reliability, delay and energy consumption of the IEEE 802.15.4 MAC layer using MCs, and showed that the performance metrics mainly depend on MAC parameters and on the collision probability. More recently, Vilajosana et al. [19] proposed an energy consumption model for the time-slotted channel hopping scheme, which is at the heart of the IEEE $802.15 .4 \mathrm{e}-$ 2012 amendment of the IEEE 802.15.4-2011 standard. Experimental validation on real devices was done, and the model was applied to different network scenarios, to understand the potential effects of several network optimizations.

These previous works focus on modeling the IEEE 802.15.4 standard, and therefore do not aim to provide generic analytical frameworks. To the best of our knowledge, only a few generic models were proposed in the literature. Vuran et al. [20] proposed a theoretical framework to exploit spatial correlation of observed events between sensor nodes on the MAC layer to reduce unnecessary data transmissions. In [21], the authors analyzed the duty-cycle, energy efficiency and latency of a handful of MAC protocols in the context of low datarate WSNs regarding various network parameters such as the network density and the transceiver. If the proposed traffic and radio models are generic, the latency and energy models are specific to each MAC, making the proposed approach hard to extend to new protocols. Asudeh et al. [22] proposed a selection framework to choose the appropriate protocol that satisfies the requirements for a given context defined by a set of input parameters. Three categories of protocols (preamble sampling, common active period and scheduled) are defined and it is assumed that protocols in the same category have similar performance characteristics. The authors defined a combined performance function that relates different metrics (delay, energy consumption... ) into a single scalar measure by scaling appropriately each metric. The aim of this performance function is to quantify the performance of each protocol to choose the most appropriate one regarding particular context and application requirements. However, the purpose of our work is not to provide a selection algorithm, but an analytical framework to evaluate different MAC schemes.

The generic framework proposed in this paper can be used to model a wide range of MAC protocols and focuses on energy consumption, latency and reliability. It is based on AMCs, and using experimental measurements, we have proved that the model provides accurate estimations in the context of low throughput applications, typical for WSNs [23].

\section{GENERIC FRAMEWORK FOR MODELING MAC PROTOCOLS}

The proposed analytical framework for modeling the energy consumption, the latency and the reliability of MAC protocols is introduced in this section. For a given protocol, a MC describing the functioning of the protocol is established. The typical modeling of a MAC protocol by a MC is illustrated by Fig. 1a. The "standby state" (STDBY) is defined as the state of the MAC when it is neither receiving nor transmitting a packet. The reception of a packet is usually preceded by a step such as the periodic check for incoming packets in preamble sampling protocols, or the asynchronous reception of a $\mathrm{WuC}$ when using WuRx. This step is called a receive wake-up and can lead to the reception of a packet. It corresponds to the R-WUP state in Fig. 1a. We call transmission process the procedure defined by the protocol to transmit a packet, and reception process the procedure defined by the protocol to receive a packet. Each of these processes consists of one or more basic steps, e.g. the transmission of a beacon, the transmission of a data frame, the reception of an acknowledgment (ACK). Some steps can possibly succeed or fail, e.g. the reception of an ACK may fail because of interference. When transmitting a packet, more than one attempt are typically allowed, and the number of allowed attempts is denoted by $N \in \mathbb{N}^{*}$. The failing of an attempt leads either to the starting of a new attempt, or to the failing of the 


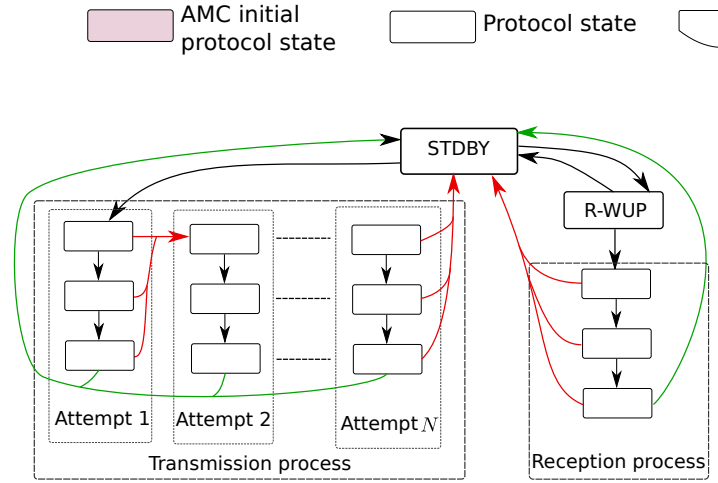

(a) Markov chain of the entire protocol.

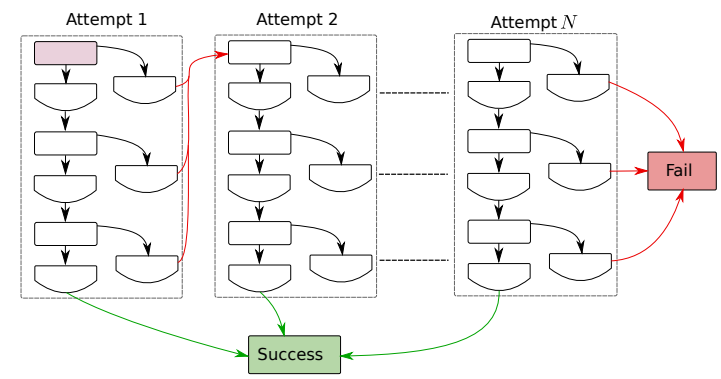

(b) $\mathrm{AMC}$ of the transmission process.
Successful process outcome

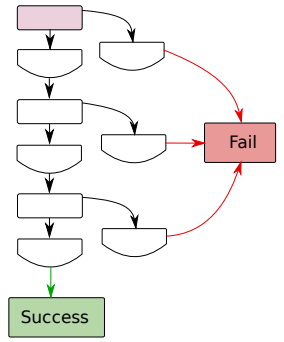

(c) AMC of the reception process.

Fig. 1: Markov chain models. States names of the transmission and reception processes are not indicated for clarity reason, as they are specific to each protocol.

transmission process if it was the last authorized attempt. The success of an attempt leads to the success of the transmission process.

In the proposed approach, the transmission and reception processes are individually modeled by two AMCs. These AMCs are constructed by extracting for each process the corresponding sub-MC of the MC modeling the entire protocol, and by defining two absorbing states, i.e. states which are impossible to leave, denoted "Success" and "Fail" and representing the two possible outcomes of the process. The AMC is termed to be "absorbed" by an absorbing state when it steps into an absorbing state. A success of the process leads to the absorption of the chain by the "Success" state and a failure of the process leads to the absorption of the chain by the "Fail" state. The modeling of the transmission and reception processes by AMCs are illustrated by Fig. 1b and Fig. 1c respectively. Three typologies of states are defined to build an AMC: protocol states, transition states and final states. Protocol states are defined by the protocol itself, and represent the steps that constitute the processes. However, to correctly evaluate the energy consumption and latency incurred by the protocol, transition states, which do not affect the behavior of the protocol, are needed to model the energy and latency cost of state transitions. The final states are the "Success" and "Fail" states that represent the possible outcomes of a packet transmission or reception process. All the states except the final ones are transient, i.e. can be left. Typically, transitions depend on the history of the traversed states. As MCs are memoryless, i.e. the probabilities of transition depend only on the current state, the state space can be augmented to ensure that the current state determines the transition probabilities. In the rest of this paper, mathematical objects (AMC, matrix, vector or scalar) associated to the packet transmission process are denoted with a "t" subscript, while mathematical objects associated to the packet reception process are denoted with a " $\mathrm{r}$ " subscript. When referring indifferently to both processes, the "b" subscript is used. The Table I gives the definition of the important symbols.

Let $C_{b}$ be an AMC modeling a packet transmission or reception process, and $\mathbf{P}_{\mathbf{b}}$ be its associated transition matrix defined by:

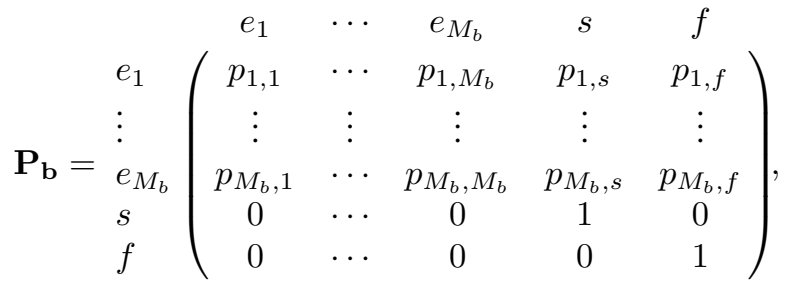

where $M_{b}$ is the number of transient states $e_{i}, i \in$ $\left\{1, \ldots, M_{b}\right\}, s$ is the final "Success" state, $f$ is the final "Fail" state, $p_{i, j} \in[0,1]$ is the transition probability from the transient state $e_{i}$ to the transient state $e_{j}, p_{i, s}$ is the transition probability from the transient state $e_{i}$ to the final state $s$ and $p_{i, f}$ is the transition probability from the transient state $e_{i}$ to the final state $f$. As $s$ and $f$ are absorbing states, the transition probabilities $p_{s, s}$ and $p_{f, f}$ take the value 1 .

\begin{tabular}{c|l}
$C_{b}$ & AMC modeling a packet transmission/reception process \\
\hline $\mathbf{P}_{\mathbf{b}}$ & Transition matrix \\
\hline $\mathbf{N}_{\mathbf{b}}$ & Fundamental matrix \\
\hline $\mathbf{n}_{\mathbf{b}}$ & Vector corresponding to the initial state row of $\mathbf{N}_{\mathbf{b}}$ \\
\hline $\mathbf{B}_{\mathbf{b}}$ & Absorption probability matrix \\
\hline$b_{b, s}$ & Probability of a successful transmission/reception \\
\hline $\mathbf{e}_{\mathbf{b}}$ & Energy cost vector \\
\hline$\overline{e_{b}}$ & Average energy cost of a packet transmission/reception \\
\hline $\bar{a}$ & Average number of transmission attempts \\
\hline $\mathbf{N}_{\mid \mathbf{a}, \mathbf{t}}$ & Conditional fundamental matrix \\
\hline $\mathbf{n}_{\mid \mathbf{s}, \mathbf{t}}$ & Vector corresponding to the initial state row of $\mathbf{N}_{\mid \mathbf{a}, \mathbf{t}}$ \\
\hline $\mathbf{l}_{\mathbf{b}}$ & Latency cost vector \\
\hline$\overline{l_{t}}$ & Expected latency of a packet transmission \\
\hline$\overline{P_{C}}$ & Average power consumption of the node \\
\hline$\lambda_{r}$ & Average packet reception rate \\
\hline$\lambda_{g}$ & Average packet generation rate \\
\hline$\lambda_{w}$ & Receive wake-up rate \\
\hline$e_{w}$ & Energy cost of a receive wake-up operation \\
\hline$l_{w}$ & Duration of a receive wake-up operation \\
\hline$P_{S B Y}$ & Power consumption of the node in STDBY mode \\
\hline
\end{tabular}

TABLE I: Table of symbols. 
Applying classical AMC results, $\mathbf{P}_{\mathbf{b}}$ can be written without loss of generality as follows [3]:

$$
\mathbf{P}_{\mathrm{b}}=\left(\begin{array}{cc}
\mathbf{Q}_{\mathrm{b}} & \mathbf{R}_{\mathrm{b}} \\
\mathbf{0}_{\mathbf{2} \times \mathbf{M}_{\mathrm{b}}} & \mathbf{I}_{2}
\end{array}\right)
$$

where $\mathbf{Q}_{\mathbf{b}}$ is a $M_{b}$-by $-M_{b}$ matrix, $\mathbf{R}_{\mathbf{b}}$ is a $M_{b}-$ by-2 matrix, $\mathbf{I}_{\mathbf{2}}$ is the identity matrix of size 2 and $\mathbf{0}_{\mathbf{2}} \times \mathbf{M}_{\mathrm{b}}$ is the $2-$ by $-M_{b}$ null matrix. The fundamental matrix of $C_{b}$ is [3]:

$$
\mathbf{N}_{\mathbf{b}}=\left(\mathbf{I}_{\mathbf{M}_{\mathbf{b}}}-\mathbf{Q}_{\mathbf{b}}\right)^{-1}
$$

The $i j$-entry of $\mathbf{N}_{\mathbf{b}}$, denoted by $n_{i, j}$, is the expected number of times the chain was in the transient state $e_{j}$ if it started in the transient state $e_{i}$ before being absorbed. It is assumed without loss of generality that the initial state of $C_{b}$ is $e_{i_{0}}$ with $i_{0} \in\left\{1, \ldots, M_{b}\right\}$, i.e. $e_{i_{0}}$ is the initial state of the transmission or reception process. Thus, only the $i_{0}^{\text {th }}$ row of the $\mathbf{N}_{\mathbf{b}}$ matrix is considered, and the vector of size $M_{b}$ corresponding to this row is denoted by $\mathbf{n}_{\mathbf{b}}$.

\section{A. Probability of a successful packet transmission or reception}

To evaluate the reliability of a protocol, the probability that a packet transmission or reception succeed, i.e. that $C_{b}$ is absorbed by the final state $s$, is considered. The absorption probability matrix denoted by $\mathbf{B}_{\mathbf{b}}$ is a $M_{b}-$ by -2 matrix in which the $i j$-entry, denoted by $b_{i, j}$, is the probability that the matrix will be absorbed by the $j^{\text {th }}$ absorbing state, which is either $s$ or $f$, if it starts in the $i^{t h}$ transient state $e_{i}$, and can be computed as follows [3]:

$$
\mathbf{B}_{\mathrm{b}}=\mathbf{N}_{\mathrm{b}} \mathbf{R}_{\mathrm{b}}
$$

As the initial state is $e_{i_{0}}$, only the $i_{0}^{\text {th }}$ row is considered and the vector of size 2 corresponding to this row is denoted by $\mathbf{b}_{\mathbf{b}}$. We denote by $b_{b, s}$ the entry of this vector corresponding to the probability that the chain $C_{b}$ is absorbed by the final state $s$, i.e. that the process successfully terminates. $b_{b, s}$ is given by:

$$
b_{b, s}=\sum_{j=1}^{M_{b}} n_{i_{0}, j} p_{j, s} .
$$

\section{B. Energy cost of a packet transmission/reception process}

Let $\mathbf{e}_{\mathbf{b}}$ be the energy costs vector, i.e. the vector of size $M_{b}$ in which the $i^{t h}$ entry is the energy cost incurred by the MAC protocol when traversing the transient state $e_{i}$. Hence, the average energy cost of a packet transmission or reception modeled by $C_{b}$ is the scalar product of $\mathbf{n}_{\mathbf{b}}$ and $\mathbf{e}_{\mathbf{b}}$ :

$$
\overline{e_{b}}=\mathbf{n}_{\mathbf{b}} \cdot \mathbf{e}_{\mathbf{b}}
$$

In (6), $\mathbf{n}_{\mathbf{b}}$ is related to the protocol functioning, while $\mathbf{e}_{\mathbf{b}}$ is related to application and hardware specifics, i.e. transmission or reception time of frames and transceiver power consumption.

\section{Average number of transmission attempts}

Let $\mathbf{c}$ be the vector of size $M_{t}$ in which the $i^{t h}$ entry takes the value 1 if $e_{i}$ corresponds to the initial state of an attempt in the AMC modeling the transmission process, 0 otherwise. Then, the expected number of transmission attempts denoted by $\bar{a}$, without considering the outcome of the transmission process, is given by:

$$
\bar{a}=\mathbf{n}_{\mathbf{t}} \cdot \mathbf{c} .
$$

\section{Latency of a packet transmission process}

We define the latency costs vector denoted by $\mathbf{l}_{\mathbf{b}}$ as the vector of size $M_{b}$ in which the $i^{t h}$ entry is the latency incurred by the MAC protocol when traversing the transient state $e_{i}$. The same reasoning as in (6) can not be applied for computing the packet transmission latency, as the scalar product of $\mathbf{n}_{\mathbf{t}}$ and $\mathbf{l}_{\mathbf{t}}$ gives the average duration of a packet transmission process without regard to its outcome. However, when the latency is evaluated, we are interested in the packet transmission duration when the transmission process succeeds. Therefore, the conditional fundamental matrix denoted by $\mathbf{N}_{\mid \mathbf{a}, \mathbf{t}}$ is introduced as the matrix in which the $i j$-entry, denoted by $n_{\mid a, i, j}$, is the expected number of times the chain was in the transient state $e_{j}$ if it started in the transient state $e_{i}$ and knowing that the chain was absorbed by the state $a \in\{s, f\}$. Moreover, it can be demonstrated that:

$$
n_{\mid a, i, j}=\frac{b_{j, a}}{b_{i, a}} n_{i, j} .
$$

Proof: Let $X_{i, j}^{(m)}$ be the random variable that takes the value 1 if the chain is at the transient state $e_{j}$ at the step $m$ if it started at the transient state $e_{i}$, and 0 otherwise. Then:

$$
\operatorname{Pr}\left(X_{i, j}^{(m)}=1\right)=q_{i, j}^{(m)},
$$

where $q_{i, j}^{(m)}$ is the $i j$-entry of the matrix $\mathbf{Q}_{\mathbf{t}}$ raised to the power $m$ denoted by $\mathbf{Q}_{\mathbf{t}}^{\mathbf{m}}$. Let $A_{i}$ be the random variable corresponding to the state, which will absorb the chain if it started at the transient state $e_{i}$. According to Bayes' theorem:

$$
\begin{aligned}
\operatorname{Pr}\left(X_{i, j}^{(m)}=1 \mid A_{i}=a\right) & \\
= & \frac{\operatorname{Pr}\left(A_{i}=a \mid X_{i, j}^{(m)}=1\right) \operatorname{Pr}\left(X_{i, j}^{(m)}=1\right)}{\operatorname{Pr}\left(A_{i}=a\right)} .
\end{aligned}
$$

Because of the Markov property:

$$
\operatorname{Pr}\left(A_{i}=a \mid X_{i, j}^{(m)}=1\right)=\operatorname{Pr}\left(A_{j}=a\right),
$$

hence, we have:

$$
\begin{gathered}
\operatorname{Pr}\left(X_{i, j}^{(m)}=1 \mid A_{i}=a\right)=\frac{\operatorname{Pr}\left(A_{j}=a\right)}{\operatorname{Pr}\left(A_{i}=a\right)} \operatorname{Pr}\left(X_{i, j}^{(m)}=1\right) \\
=\frac{b_{j, a}}{b_{i, a}} q_{i, j}^{(m)}, \quad
\end{gathered}
$$

using the notation of the absorption probabilities matrix $\mathbf{B}_{\mathbf{b}}$. The expected number of times the chain was in the transient 
state $e_{j}$ in the first $m$ steps given that it started in the transient state $e_{i}$ and that it was absorbed by the state $a$ is:

$$
\begin{aligned}
\mathbb{E}\left(X_{i, j}^{(0)}+\cdots+X_{i, j}^{(m)} \mid\right. & \left.A_{i}=a\right) \\
= & \frac{b_{j, a}}{b_{i, a}}\left(q_{i, j}^{(0)}+\cdots+q_{i, j}^{(m)}\right),
\end{aligned}
$$

because of the linearity of the expectancy. Hence, when $m$ goes to infinity:

$$
\begin{aligned}
n_{\mid a, i, j} & =\lim _{m \rightarrow+\infty} \mathbb{E}\left(X_{i, j}^{(0)}+\cdots+X_{i, j}^{(m)} \mid A_{i}=a\right) \\
& =\lim _{m \rightarrow+\infty} \frac{b_{j, a}}{b_{i, a}}\left(q_{i, j}^{(0)}+\cdots+q_{i, j}^{(m)}\right)=\frac{b_{j, a}}{b_{i, a}} n_{i, j} .
\end{aligned}
$$

where the last equality holds because [3]:

$$
\mathbf{N}=\sum_{k=0}^{\infty} \mathbf{Q}_{\mathbf{b}}{ }^{k},
$$

where $\mathbf{Q}_{\mathbf{b}}{ }^{0}=\mathbf{I}_{\mathbf{M}_{\mathbf{b}}}$.

The conditional fundamental matrix $\mathbf{N}_{\mid \mathbf{s}, \mathbf{t}}$ is such that the $i j$-entry is the expected number of times the chain was in the transient state $e_{j}$ if it started in the transient state $e_{i}$ knowing that the packet transmission succeeds. As the initial state is assumed to be $e_{i_{0}}$, only the vector corresponding to the $i_{0}^{t h}$ row and denoted by $\mathbf{n}_{\mid \mathbf{s}, \mathbf{t}}$ is considered. Hence, the expected latency incurred by the transmission of a packet is:

$$
\overline{l_{t}}=\mathbf{n}_{\mid \mathbf{s}, \mathbf{t}} \cdot \mathbf{l}_{\mathbf{t}},
$$

where $\mathbf{l}_{\mathbf{t}}$ is the latency cost vector of the transmission process.

\section{E. Average power consumption}

Knowing the expected energy costs of a packet transmission and a packet reception, respectively denoted by $\overline{e_{t}}$ and $\overline{e_{r}}$ and computed using (6), the average power consumption, denoted by $\overline{P_{C}}$, incurred by communications can be computed as follows:

$$
\begin{aligned}
\overline{P_{C}}= & \bar{a} \lambda_{r} \overline{e_{r}}+\left(\lambda_{g}+b_{t, s} \lambda_{r}\right) \overline{e_{t}}+\lambda_{w} e_{w}+\left(1-\bar{a} \lambda_{r}\left(\mathbf{n}_{\mathbf{r}} \cdot \mathbf{l}_{\mathbf{r}}\right)\right. \\
& \left.-\left(\lambda_{g}+b_{t, s} \lambda_{r}\right)\left(\mathbf{n}_{\mathbf{t}} \cdot \mathbf{l}_{\mathbf{t}}\right)-\lambda_{w} l_{w}\right) P_{S B Y},
\end{aligned}
$$

assuming that a node forwards all packets that are successfully received. In (17), $\lambda_{r}$ and $\lambda_{g}$ are respectively the average packet reception and local packet generation rate. $b_{t, s}$ is the probability that a packet transmission succeeds and is computed given by (5). $\lambda_{w}$ is the receive wake-up rate, and $e_{w}$ and $l_{w}$ are respectively the energy cost and the duration of a single receive wake-up operation. The scalar products $\mathbf{n}_{\mathbf{r}} \cdot \mathbf{l}_{\mathbf{r}}$ and $\mathbf{n}_{\mathbf{t}} \cdot \mathbf{l}_{\mathbf{t}}$ correspond to the duration of a packet reception and packet transmission process respectively, without regard to the outcome of the processes. Finally, $P_{S B Y}$ corresponds to the power consumption of the node when the MAC is in the STDBY state.

In (17), the first term corresponds to the power consumption due to packets reception, and the second term to the power consumption incurred by packets transmission. The third term accounts for the power consumption due to the receive wakeup operation (periodic listening to the channel or beacon transmission, WuCs reception...), and the final term accounts for the power consumption of the node in sleep state.

\section{F. Constructing the AMCs transition matrices}

The construction of the AMCs requires the calculation of the transition probabilities, which depend both on the protocol functioning and on the frame failure probabilities, i.e. the probability of frame transmission and reception failures. In order to compute the frame failure probabilities, analytical models which focus on the wireless channel and interferences [24] can be used. Combining the framework proposed in this work with such models allows the evaluation of the MAC protocols regarding the scalability and channel quality. In the rest of the paper, the frame failure probability is denoted by $p_{f}$, which can take different values for different frames. Hence, when constructing an AMC modeling a packet transmission or reception process, the states corresponding to a frame transmission or reception lead to a failure with a probability $p_{f}$, and to a success with a probability $1-p_{f}$.

\section{Case study: Modeling PW-MAC using the PROPOSED FRAMEWORK}

In this section, PW-MAC [6], a receiver-initiated protocol that focuses on low energy consumption for both the sender and the receiver, is modeled using the proposed framework. PW-MAC was proposed by the same authors that introduced RI-MAC [7], to reduce the power consumption of the latter. Through this case study, the different stages required to apply the proposed framework to a particular MAC are illustrated. First, the AMCs modeling the transmission and reception processes are established. Then, the energy and latency cost vectors are set. Two different approaches are presented to set the energy and latency cost vectors. The first one relies solely on analytical estimations, while the second combines analytical estimations with measurements obtained by microbenchmarks to obtain accurate values of the energy and latency overhead incurred by the software and hardware. By comparing the accuracy of the model achieved by each approach, the benefits enabled by the second approach are shown.

\section{A. Building the AMCs}

The first step for modeling a particular MAC protocol using the proposed framework is to build the two AMCs describing the packet transmission and the packet reception processes. PW-MAC is based on the duty-cycling approach, in which the node keeps its transceiver in sleep state most of the time, and regularly switches it on to check for incoming packet. The proportion of time during which the node is active is called the duty-cycle. Because PW-MAC is a receiver-initiated protocol, each node regularly sends beacon indicating that it is ready to receive. After each beacon sending, it listens to the channel for incoming data packet. If no packet is detected on the channel, the node goes back to sleep. The time interval 


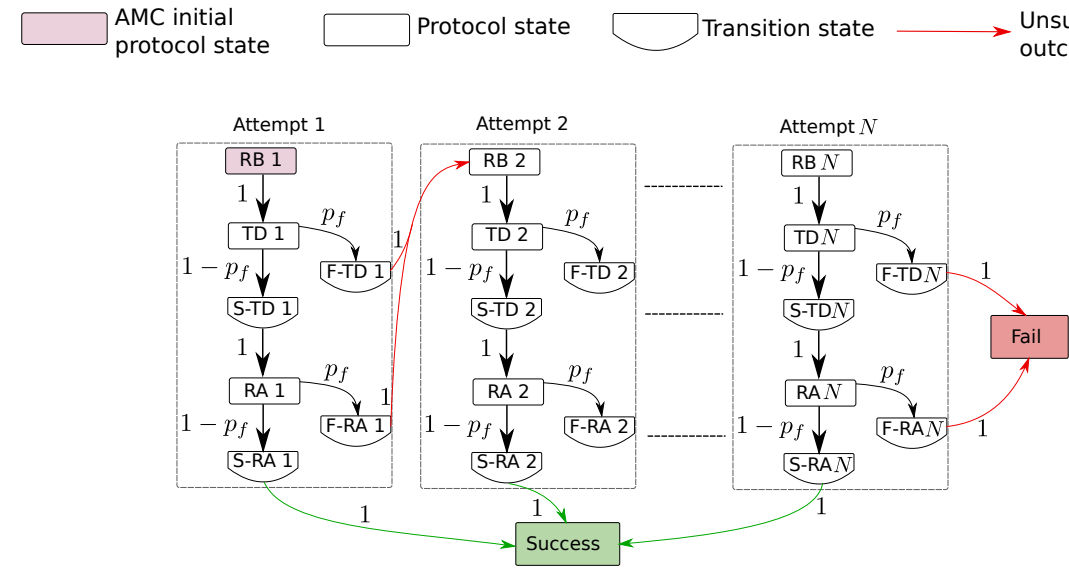

(a) AMC modeling the transmission process of PW-MAC.
Unsuccessful process outcome Successful process outcome

Fig. 3: AMCs modeling the transmission and reception processes of PW-MAC.
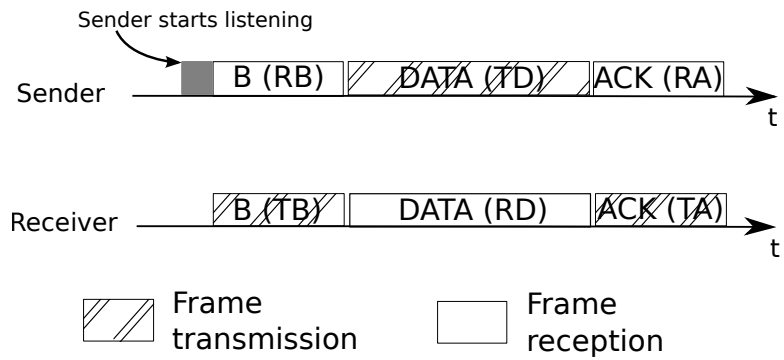

Fig. 2: Packet transmission using PW-MAC. For each frame transmission or reception, the corresponding state name is indicated in brackets.

between two beacon sendings is computed using a pseudorandom generator, avoiding nearby nodes to wake up at the same time repeatedly. A node learns the wake-up schedule of its forwarders, and when it needs to send a packet, it wakes up just before the receiving node sends a scheduled beacon. After receiving the beacon from the forwarder, it sends the data frame, and then listens to the ACK frame. PW-MAC operation is shown by Fig. 2. In this figure, the protocol state names associated to each frame sending or receiving are indicated in brackets.

1) Packet transmission process AMC: The transmission process of PW-MAC is modeled by the AMC shown by Fig. 3a. Each protocol state corresponds to the sending or receiving of a frame. When a node needs to transmit a packet, it first listens to the channel until it receives a beacon. Therefore, the protocol states corresponding to this operation, named $\mathrm{RB} i$ where $i \in\{1, \ldots, N\}$ is the attempt number, only has one outcome as opposite to the other protocol states TD $i$ and RA $i$, which possibly finish by a success or a failure. Transition states were added to take into account the energy and latency costs incurred by each possible outcome (success or failure) of the TD $i$ and RA $i$ protocol states. For example, if the TD $i$ state fails, i.e. the transmission of the data frame fails at the $i^{t h}$ attempt, then the node will still listen for an ACK, which will incur energy consumption and latency. These energy and latency costs are accounted by the F-TD $i$ transition state. As there are $M_{t}=7$ transient states, the associated transition matrix is the $(7 N+2)-$ by $-(7 N+2)$ matrix is defined by:

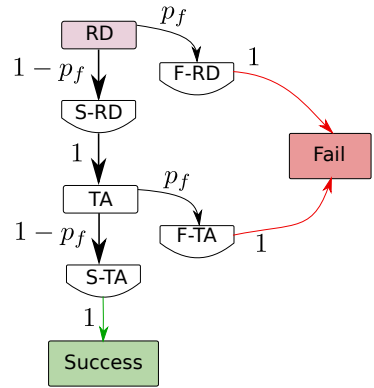

(b) AMC modeling the reception process of PWMAC.

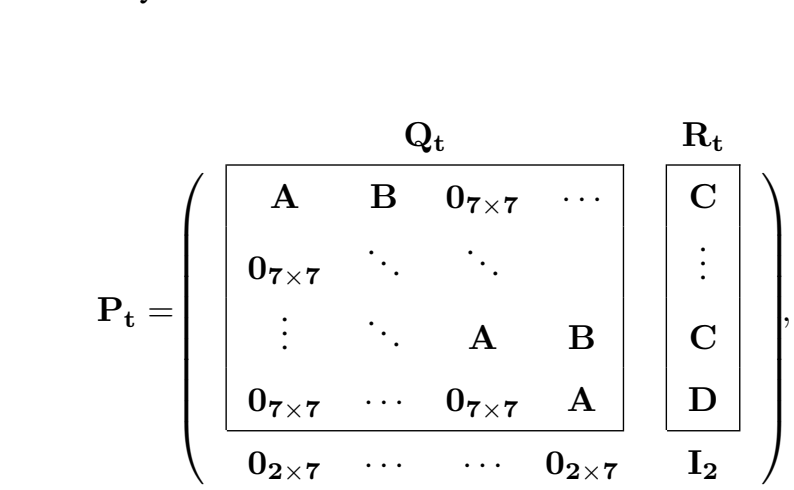

where $\mathbf{A}$ is the $7-$ by -7 matrix corresponding the to the intraattempt transitions:

\begin{tabular}{|c|c|c|c|c|c|c|c|}
\hline & $\mathrm{RB} i$ & TD $i$ & RA $i$ & S-TD $i$ & F-TD $i$ & S-RA $i$ & F-RA $i$ \\
\hline $\mathrm{RB} i$ & 0 & 1 & 0 & 0 & 0 & 0 & 0 \\
\hline TD $i$ & 0 & 0 & 0 & $1-p_{f}$ & $p_{f}$ & 0 & 0 \\
\hline RA $i$ & 0 & 0 & 0 & 0 & 0 & $1-p_{f}$ & $p_{f}$ \\
\hline $\mathbf{A}=\mathrm{S}-\mathrm{TD} i$ & 0 & 0 & 1 & 0 & 0 & 0 & 0 \\
\hline F-TD $i$ & 0 & 0 & 0 & 0 & 0 & 0 & 0 \\
\hline S-RA $i$ & 0 & 0 & 0 & 0 & 0 & 0 & 0 \\
\hline F-RA $i$ & 0 & 0 & 0 & 0 & 0 & 0 & 0 \\
\hline
\end{tabular}

where $i \in\{1, \ldots, N\}$. Similarly, $\mathbf{B}$ is the $7-$ by -7 matrix corresponding to the transitions between an attempt and the next attempt:

\begin{tabular}{|c|c|c|c|c|c|}
\hline & & $\mathrm{RB} i+1$ & TD $i+1$ & $\cdots$ & F-RA $i+1$ \\
\hline & $\mathrm{RB} i$ & 0 & 0 & $\cdots$ & 0 \\
\hline & TD $i$ & 0 & 0 & $\ldots$ & 0 \\
\hline & RA $i$ & 0 & 0 & $\cdots$ & 0 \\
\hline $\mathbf{B}=$ & S-TD $i$ & 0 & 0 & $\cdots$ & 0 \\
\hline & F-TD $i$ & 1 & 0 & $\ldots$ & 0 \\
\hline & S-RA $i$ & 0 & 0 & $\cdots$ & 0 \\
\hline & F-RA $i$ & 1 & 0 & $\ldots$ & 0 \\
\hline
\end{tabular}


where $i \in\{1, \ldots, N-1\}$. $\mathbf{C}$ is the matrix corresponding to the transitions between the non-last attempts and the final states:

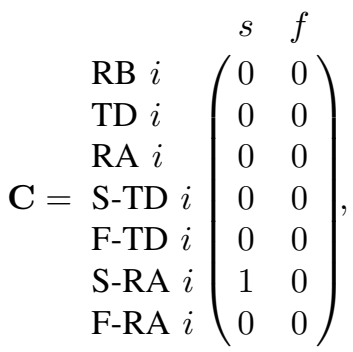

where $i \in\{1, \ldots, N-1\}$. Finally, $\mathbf{D}$ is the matrix corresponding to the transitions between the last attempt and the final states:

$\left.\begin{array}{l|ll} & \multicolumn{1}{c}{s} & f \\ & \operatorname{RB} N \\ \operatorname{TD} N & 0 & 0 \\ \operatorname{RA} N & 0 & 0 \\ \operatorname{R}-T D & 0 & 0 \\ \text { F-TD } N & 0 & 0 \\ \text { S-RA } N & 0 & 1 \\ \text { F-RA } N & 0 \\ 0 & 1\end{array}\right)$.

2) Packet reception process $A M C$ : PW-MAC requires each node to wake up regularly to send a beacon indicating that it is ready to receive a packet. The node then listens to the medium for incoming packet, and if no preamble is detected, it returns to sleep after a short time. Otherwise, it continues to listen for the incoming packet. This operation is modeled by the RWUP state of the MC modeling the protocol, and is therefore not considered by the AMC modeling the packet reception process. The initial state of the AMC modeling the packet reception process is the state corresponding to a data frame reception, as shown by Fig. 3b. In this AMC, the state RD corresponds to a data frame reception, and the state TA to an acknowledgment transmission. Transition states were added to take into account the energy and latency costs incurred by each state possible outcome (success or failure). The corresponding transition matrix is the following $8-$ by -8 matrix:

$$
\mathbf{P}_{\mathrm{r}}=\left(\begin{array}{cc}
\mathrm{Q}_{\mathrm{r}} & \mathbf{R}_{\mathrm{r}} \\
\mathbf{0}_{2 \times 6} & \mathbf{I}_{2}
\end{array}\right),
$$

where $\mathbf{Q}_{\mathbf{r}}$ is the 6-by-6 matrix that corresponds to transitions between non-final states:

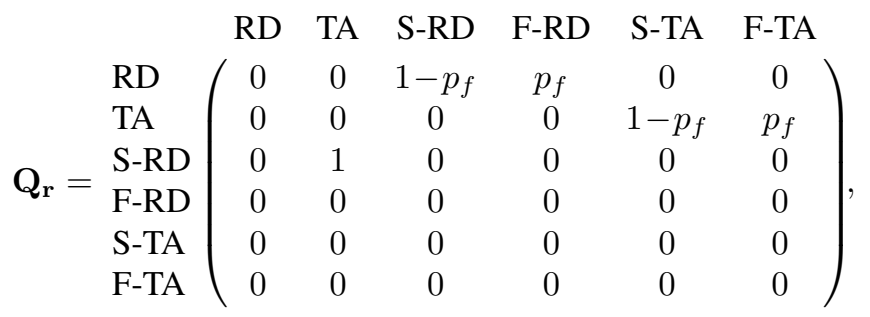

and $\mathbf{R}_{\mathbf{r}}$ is the $6-$ by -2 matrix that corresponds to transitions between the non-final states and the two final states:

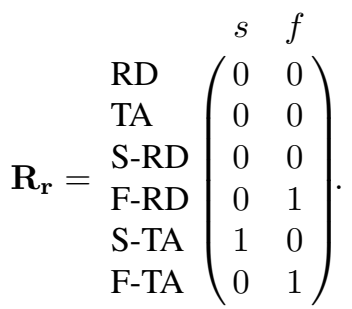

\section{B. Setting the energy and latency cost vectors}

The proposed framework requires the energy cost and latency cost vectors, respectively denoted by $\mathbf{e}_{\mathbf{b}}$ and $\mathbf{l}_{\mathbf{b}}$, to be set carefully. When a node has to transmit a packet using PWMAC, it first listens continuously until it receives a beacon. This operation corresponds to the RB $i$ states of the AMC modeling the packet transmission process. It is assumed that the probability of a frame failure is $p_{f}$, therefore the number of failures before a beacon is successfully received, denoted by $X$, is a discrete random variable that follows a geometric distribution of parameter $1-p_{f}$. The expected number of beacon reception failures before the first beacon reception success is thus:

$$
\mathbb{E}[X]=\frac{p_{f}}{1-p_{f}} .
$$

The average rate of beacon sending by a node is $\lambda_{w}$, and the expected listening time before a successful beacon reception starts, denoted by $\mathbb{E}[L]$, is therefore:

$$
\mathbb{E}[L]=\frac{p_{f}}{\lambda_{w}\left(1-p_{f}\right)},
$$

which was accounted in the latency cost of the RB $i$ state of the transmission process.

Focusing on the energy and latency costs incurred by the transmission and reception of frames (beacon, data and ACK), the size of a frame in bits is denoted by $S$, and the transmission bit-rate in bps is denoted by $R$. When a protocol is implemented on a real platform, energy and latency overhead due to hardware and software overhead can incur as a consequence of radio setup, turn around... Therefore, the latency incurred by a frame transmission/reception is:

$$
l=\frac{S}{R}+l_{o v h}
$$

where $l_{\text {ovh }}$ is the hardware/software overhead. Moreover, the energy cost incurred by a frame transmission/reception is:

$$
e=\frac{S}{R} P_{C}+e_{o v h}
$$

where $P_{C}$ is the power consumed by the node, and takes different values depending on whether the node is transmitting or receiving, and $e_{\text {ovh }}$ is the hardware/software energy overhead. The energy cost and latency cost incurred by the transmission/reception of each frame (beacon, data and ACK) were calculated using the appropriate values of $S, R$ and $P_{C}$.

Microbenchmarks were performed to measure accurately the energy and latency costs (including the overhead) of a packet transmission, a packet reception, and a scheduled 


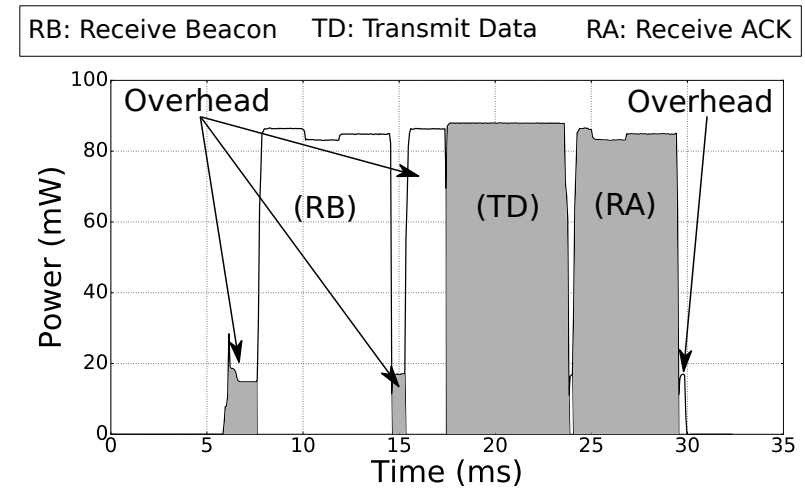

Fig. 4: Power consumption trace of a packet transmission using PWMAC.

wake-up. The measurement results are shown by Fig. 4 for the packet transmission process. These measurements were done using a Keysight N6705B DC power analyzer, with the PowWow platform [25]. PowWow is a modular platform able to use a wide range of transceivers. In this work, the Texas Instrument (TI) CC1120 transceiver was considered, which consumes $34 \mathrm{~mA}$ when transmitting at $+10 \mathrm{dBm}$, and $22 \mathrm{~mA}$ when receiving, according to the datasheet. On Fig. 4, the different steps of the packet transmission process can be seen, and the corresponding states are indicated. This figure shows that hardware and software overhead is introduced at the beginning and at the end of the processes, as well as between frames sendings and reception. These additional energy and latency costs were taken into account when setting the energy and latency cost vectors, and similar measurements were done for the reception process and the regular wakeups. To illustrate the importance of considering the hardware and software overhead when setting the cost vectors, measurements of the power consumption and latency using the experimental setup described in Section V-B were done. Measurements were done for different average values of $\lambda_{w}$, and compared to the analytical estimation obtained using the proposed framework. For both the latency and the power consumption, two analytical estimations were realized: one using cost vectors that do not take into account the hardware and software overhead, i.e. $e_{o v h}$ and $l_{\text {ovh }}$ were set to zero, qualified as "naive", and one that considers this overhead using the microbenchmark measurements qualified as "hybrid", as it uses both analytical estimation and experimental measurements from the microbenchmarks. Fig. 5 shows the two estimations as well as the obtained measurements. As it can be seen, the proposed framework is accurate regarding the latency both using the "naive" and the "hybrid" approaches. However, regarding the power consumption, the "hybrid" approach performs significantly better than the "naive" one. This is because most of the power consumption of PW-MAC is due to the scheduled wake-ups. Therefore having accurate measurement of the energy cost of this operation is essential to achieve accurate estimation of the power consumption. These results show the benefits of the "hybrid" approach compared to the "naive" one to achieve accurate estimation using the proposed framework.

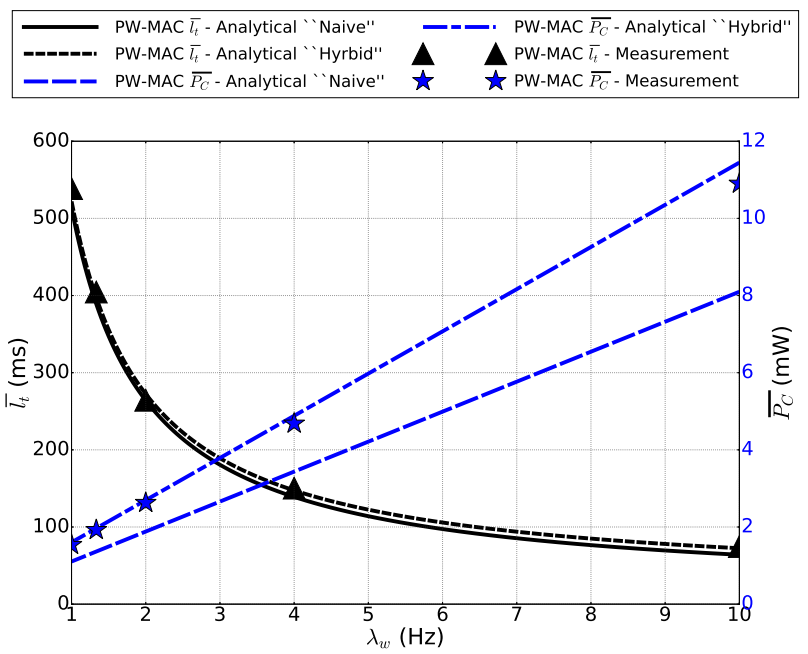

Fig. 5: Comparison of the "naive" setting of the cost vectors and "hybrid" setting.

\section{COMPARISON OF MAC PROTOCOLS}

The purpose of the framework introduced in Section III is to enable comparison between a wide range of MAC protocols, possibly based on different paradigms (preamble sampling, TDMA...). Moreover, this framework allows the evaluation of new paradigms. To illustrate how the proposed framework can be used to evaluate new approaches, we evaluate in this section a pure-asynchronous communication scheme, enabled by the emergence of ULP WuRx [9]-[11]. We first provide a brief presentation of ULP WuRx to motivate the usage of these new devices and the energy efficient MAC paradigm they enable. Then, the latency-power consumption trade-off is evaluated for various MAC protocols, including one leveraging ULP WuRx. Finally, the reliability of these MAC protocols regarding the packet failure probability is evaluated.

\section{A. Presentation of ULP WuRx}

When the communication traffic is low (around a few packets per minute), traditional pseudo-asynchronous schemes based on preamble sampling and relying on duty-cycling are used [2]. In these approaches, each node is most of the time in sleep state, and regularly wakes up to check the channel for incoming packet. As nodes frequently wake up while no incoming packet are pending, idle listening is usually a significant source of energy waste. Therefore, when setting the wake-up rate, i.e. the rate at which nodes wake up to check for incoming packet, a compromise is made between power consumption and latency.

Emerging ULP WuRx enable pure-asynchronous communication. In this approach, each node is equipped with an ULP WuRx, which allows a continuous channel monitoring while consuming orders of magnitude less power than traditional transceivers [8]. When a WuC is detected, the ULP WuRx wakes up the node MCU or other sleeping subsystems. Using these devices, nodes do not need to regularly turn on their main transceiver to avoid deafness, and idle listening is thus minimized. Moreover, some state-of-the-art ULP WuRx provide computational capabilities [26], [27], and are therefore able to 


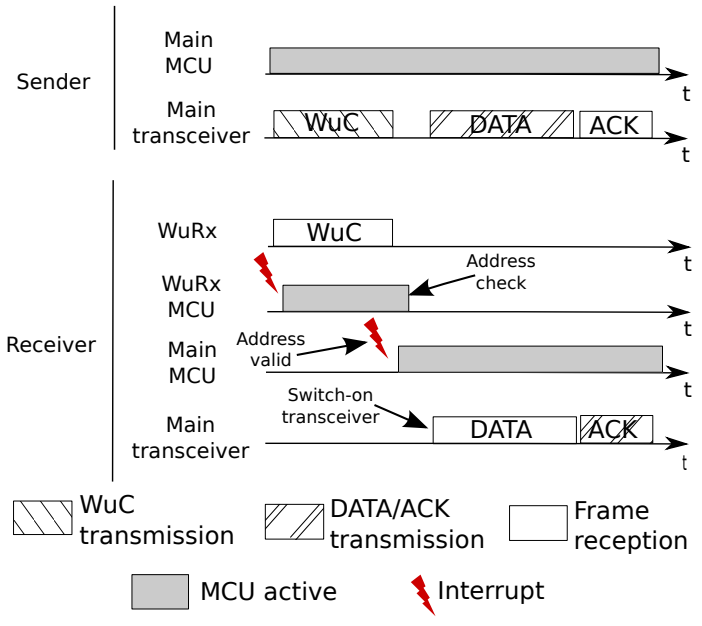

Fig. 6: Illustration of a packet transmission using TI-WuR.

process the received data autonomously in an energy efficient manner. This feature makes possible a $\mathrm{WuC}$ to wake up only a specific node by performing address matching on the ULP WuRx, but it also allows the ULP WuRx to take action without waking up the main MCU. The main drawback of state-of-theart ULP WuRx come from their low sensitivity and their low bit-rate compared with traditional WSN transceivers [8], [26], [27], which is the price of ULP consumption. Consequently, WuCs are sent at higher transmission power and lower bit-rate than the other frames received by the main radio to achieve the same transmission range, making the sending of $\mathrm{WuCs}$ energetically expensive.

\section{B. Evaluation setup}

The ULP WuRx designed and developed in [26] was used for the experimental evaluation. This ULP WuRx works in the $868 \mathrm{MHz}$ frequency bands, and receives data with OnOff Keying (OOK) modulation at a bit-rate of $1 \mathrm{kbps}$. The sensitivity in these conditions was measured to be $-55 \mathrm{dBm}$. This ULP WuRx provides computational capabilities due to the Microchip PIC12LF1552 ULP MCU that it embeds. The power consumption of the whole ULP WuRx was measured to be $1.83 \mu \mathrm{W}$ in the always-on listening mode, and $284 \mu \mathrm{W}$ when receiving and processing data.

To evaluate the pure-asynchronous approach, TI-WuR, a transmitter-initiated MAC protocol leveraging ULP WuRx, was modeled using the proposed framework, and following the procedure described in Section IV. A packet transmission using TI-WuR is illustrated by Fig. 6. The sender first sends a WuC containing the address of the receiver using OOK modulation at $1 \mathrm{kbps}$. The analog front-ends of the ULP WuRx of its neighbors detect the activity on the channel, and trigger an interrupt to wake up the MCU embedded in the ULP WuRx. The ULP WuRx MCU then reads the incoming data, and performs address matching. If the address embedded in the $\mathrm{WuC}$ matches the node address, the ULP WuRx MCU triggers an interrupt to wake up the main MCU. The main MCU then switches-on the main transceiver to receive the data frame from the sender. The data frame is sent using standard IEEE 802.15.4 modulation schemes and bit-rates, and using the standard IEEE 802.15.4 frame structure which provides Cyclic Redundancy Check (CRC) codes for error detection. Finally, an ACK frame is sent by the receiver to acknowledge the reception of the data frame.

In addition to TI-WuR, RI-WuR, a receiver-initiated protocol leveraging WuRx, was also implemented. Using RI$\mathrm{WuR}$, each node periodically broadcasts a $\mathrm{WuC}$ to inform neighboring nodes that it is ready to receive. When a node receives a $\mathrm{WuC}$, two scenarios are possible. If the node has no packet to send to the node that broadcasts the $\mathrm{WuC}$, its $\mathrm{WuRx}$ simply ignores the WuC. Otherwise, the node is awaken, and the data transmission starts using the main transceiver in the same way that with TI-WuR. TI-WuR and RI-WuR are compared to the PW-MAC, X-MAC and CSMA/CA protocols. PW-MAC is an improvement of RI-MAC, proposed by the same authors, which focuses on low energy consumption. The details of PW-MAC modeling using the proposed framework are given in Section IV. X-MAC is a well-known transmitterinitiated protocol in which the receiver periodically wakes up to listen to the channel for a short time. When a node needs to send a packet, it first sends short preambles with the target address. Once the receiver detects a short preamble frame with its address, it sends an early ACK, and the transmitter then sends the data frame. The CSMA/CA scheme was also evaluated. CSMA/CA is used by the IEEE 802.15.4 MAC standard in beaconless mode, and requires the node to be continuously listening to the channel. Therefore, when a node needs to send a data frame, it sends the data frame directly to the addressee node, possibly after a random backoff. The reception of the data frame is acknowledged by an ACK frame. If this approach incurs high power consumption, it provides benchmark values for latency. Similarly to PW-MAC, TI-WuR, RI-WuR, CSMA/CA and X-MAC were modeled using the proposed framework and following the procedure illustrated in Section IV.

These five protocols were implemented on a testbed of PowWow platforms. Each PowWow node was equipped with a TI CC1120 transceiver, and, when TI-WuR or RI-WuR were evaluated, each node was also equipped with an instance of the ULP WuRx presented above. The transmission power of the WuCs was $+10 \mathrm{dBm}$, allowing a range up to $25 \mathrm{~m}$ using $+3 \mathrm{dBi}$ antennas for both transmitters and receivers, while the transmission power of the non-WuC frames was $-6 \mathrm{dBm}$ as it was found experimentally to be the minimal power required to achieve the same range. Moreover, the size of the data payload frame was 14B. Each measurement was done on a node that was receiving packets at the rate $\lambda_{r}=0.10 \mathrm{~Hz}$ (not considering the outcome of the reception process), locally generating packets at the rate $\lambda_{g}=0.10 \mathrm{~Hz}$, and transmitting both the generated and the received packets.

\section{Beyond the latency-power consumption trade-off}

Fig. 7 exposes the latency and the power consumption incurred by the evaluated protocols. Both analytical and experimental results are shown. For the analytical evaluation, $p_{f}$ was set to 0 as this value lead to the closest fit with the experimental measurements. Concerning RI-WuR, PW-MAC and X-MAC, latency and power consumption were computed 


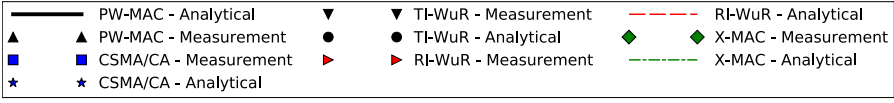
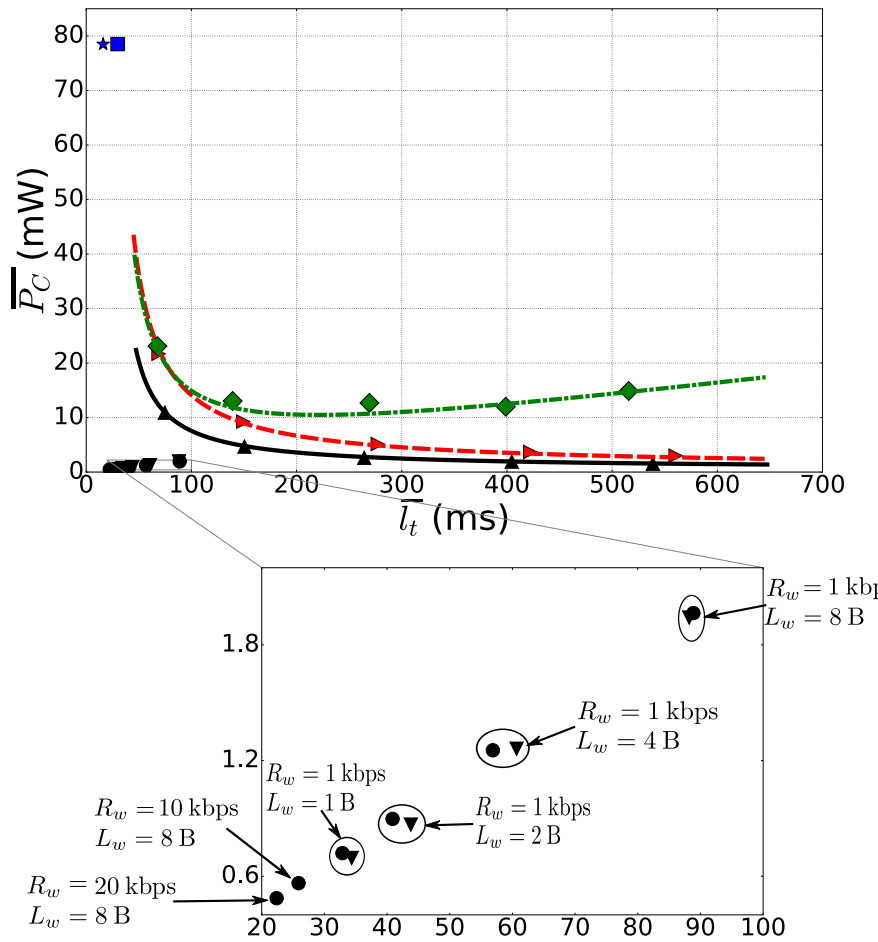

Fig. 7: Power consumption and latency of the five evaluated protocols.

with the proposed framework for values of the average wakeup rate $\lambda_{w}$ in the range $[1,20] \mathrm{Hz}$. Moreover, experimental measurements of these two quantities are shown for values of $\lambda_{w}$ of $1,1.3,2,4$ and $10 \mathrm{~Hz}$. In the case of TI-WuR, results for different values of the bit-rate at which WuCs are sent, denoted by $R_{w}$, and different values of WuCs size, denoted by $L_{w}$, are exposed. Regarding CSMA/CA, only one couple of points (analytical and experimental) is shown as the protocol has no tuning parameters. It can be seen that the model fits well the numerical results for the three evaluated protocols.

These results show that a trade-off between the latency and the power consumption must be made when using RI-WuR and PW-MAC, and this trade-off is set by the wake-up rate $\lambda_{w}$. Indeed, high values of $\lambda_{w}$ incur low power consumption at the cost of high latency, while low values of $\lambda_{w}$ lead to low latency at the cost of high power consumption. Therefore, minimizing both latency and power consumption using this approach is not straightforward. Regarding X-MAC, an optimal value of $\lambda_{w}$ permits the minimization of the power consumption. However, this optimal value depends on both $\lambda_{r}$ and $\lambda_{g}$, which makes this optimization process difficult. When using X-MAC, choosing low values of $\lambda_{w}$ allows low latencies at the cost of high power consumption, similarly to PW-MAC and RI-WuR. However, as X-MAC sends short preambles before each packet transmission, using high values of $\lambda_{w}$ incurs high power consumption as the overhead incurred by the preamble sending becomes significant. PW-MAC and RI-WuR do not suffer from this drawback. Indeed, with PW-MAC the transmitter is synchronized with the receiver, and RI-WuR uses WuRx to avoid the need of preamble sending. CSMA/CA minimizes the latency as nodes are always listening: no synchronization nor $\mathrm{WuC}$ sending is required. However, this comes at the cost of high power consumption.

TI-WuR allows significantly lower latency and power consumption compared to the other considered protocols as it can be seen on Fig. 7. As WuCs are sent at higher transmission power and lower bit-rate than the other frames, decreasing the WuCs transmission time leads to lower latency and power consumption. Therefore, for $R_{w}=1 \mathrm{kbps}$, smaller $\mathrm{WuC}$ sizes lead to better performance, as shown by Fig. 7 for WuCs sizes ranging from $1 \mathrm{~B}$ to $8 \mathrm{~B}$. However, reducing the $\mathrm{WuCs}$ size is not always a solution as it implies reducing the amount of information that WuCs embed. Therefore, a more promising solution is to increase the bit-rate of ULP WuRx. As the ULP WuRx device used for experimentation is optimized for a bitrate of $1 \mathrm{kbps}$, the evaluation of TI-WuR for values of $R_{w}$ higher than $1 \mathrm{kbps}$ was only done analytically, and Fig. 7 shows the latency and power consumption incurred by TIWuR when $L_{w}=8 \mathrm{~B}$ and $R_{w}$ equals $10 \mathrm{kbps}$ and $20 \mathrm{kbps}$. TI-WuR achieves a latency of $25.9 \mathrm{~ms}$ when $R_{w}=10 \mathrm{kbps}$, and $22.0 \mathrm{~ms}$ when $R_{w}=20 \mathrm{kbps}$, while CSMA/CA achieves a latency of $16.2 \mathrm{~ms}$, but at the cost of a power consumption 130 times higher.

These results show the benefits of the pure-asynchronous approach enabled by ULP WuRx. Trade-off between the power consumption and the latency are no longer required in the context of low data-rate WSNs, as packet exchanges are done without requiring regular wake-ups as with preamble sampling protocols, or continuous listening of the main transceiver as with CSMA/CA. Performance of current schemes can be improved by increasing the bit-rate of ULP WuRx, however this come at the cost of a trade-off with the range and the power consumption of the ULP WuRx device [26].

\section{Reliability evaluation}

This section focuses on the impact of the frame failure probability $p_{f}$ on the performance of three MAC protocols, TIWuR, PW-MAC and CSMA/CA. TI-WuR and PW-MAC were chosen as they present the best performance, while CSMA/CA was chosen as it gives benchmark values for the latency. High values of $p_{f}$ can be caused by interferences or collisions, e.g. due to dense networks or poor channel quality. As it is difficult to control the frame failure probability experimentally, the impact of this parameter was only analytically evaluated. Fig. 8a shows the impact of $p_{f}$ on $\overline{P_{c}}$ when $\lambda_{w}=4 \mathrm{~Hz}$, $R_{w}=1 \mathrm{kbps}$ and $L_{w}=1 \mathrm{~B}$. It can be seen that while $p_{f}$ has a low impact on the average power consumption incurred by TIWuR and CSMA/CA, it has a strong impact on the average power consumption incurred by PW-MAC. Indeed, when a node sends a packet using PW-MAC to a forwarder node, the sending node first wakes up just before the forwarder sends its scheduled beacon. If no beacon is received, then the sending node assumes that the synchronization with the forwarder node was lost, keeping its transceiver active until a beacon from the forwarder node is received to resynchronize. Therefore, a high frame failure probability causes frequent resynchronization activities and therefore significantly increases the power consumption. This unwanted effect also leads to high packet 


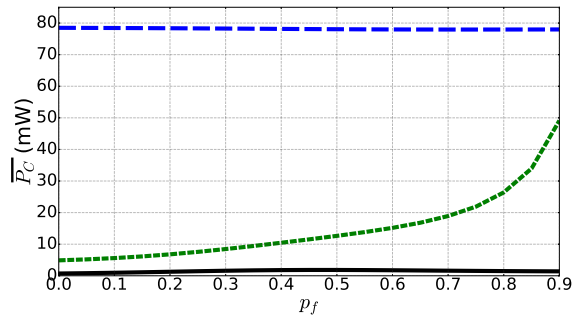

(a) $\overline{P_{C}}$ as a function of $p_{f}$.

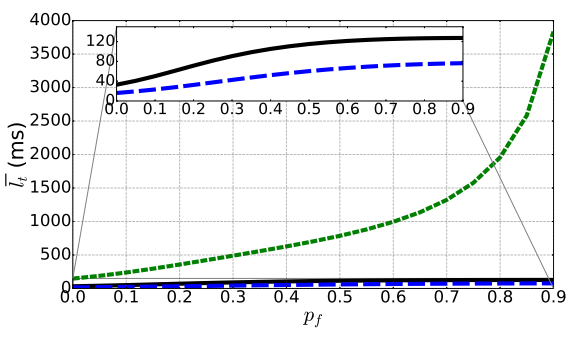

(b) $\overline{l_{t}}$ as a function of $p_{f}$.

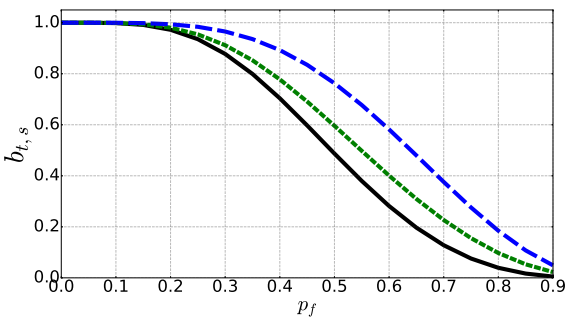

(c) $b_{t, s}$ as a function of $p_{f}$.

Fig. 8: Average power consumption, average transmission latency and probability of transmission success regarding the frame failure probability.

transmission latency when $p_{f}$ becomes high as shown by Fig. 8b, as the sending node waits for a valid beacon from the forwarder node. On the other hand, when an attempt to transmit a packet fails with TI-WuR or CSMA/CA, the sending node makes a new attempt until the transmission succeeds or exceeds a predefined number of re-transmissions (set to 4 in this work), each re-transmission being preceded by a random backoff. Nonetheless, the functioning of PWMAC leads to higher probability of successfully delivering the packet compared to TI-WuR as shown by Fig. 8c. However, the difference becomes significant only when the probability of frame failure is higher than $20 \%$. CSMA/CA is the more reliable protocol, as only two frames must successfully be transmitted for the communication to be successful (the data frame and the ACK), while three frames must be successfully transmitted for TI-WuR and PW-MAC.

\section{Discussion AND CONCLUSION}

In this paper, a new generic framework for modeling MAC protocols was presented. This framework is based on absorbing Markov chains, and focuses on energy consumption, latency and reliability. The steps required to model a specific MAC protocol using the proposed framework were illustrated using the PW-MAC protocol, which aims to improve energy efficiency for both the receiver and the sender. Moreover, experimental measurements were performed to accurately set the energy and latency parameters required by the model, and to validate the framework.

While using the proposed framework to evaluate novel schemes, several lessons were learned about the potential and the limitations of the framework. Firstly, the energy and latency strongly depend on the hardware and the implementation, and therefore measurements are required if high accuracy is desired. In this work, microbenchmarks were used to get accurate values of both the latency and energy consumption incurred by each state. This can be seen as a limitation of the proposed framework as it requires measurements on real hardware. However, if one is interested only by the trends of the protocols, highly accurate estimation of the energy and latency is not necessary.

Another potential issue is the construction of the transition matrices, which requires the calculation of the transition probabilities. These probabilities depend on both the protocol algorithm and the frame failure probability. Computing realistic value of the frame failure probability for a given precise context may be difficult, as it depends on the channel state. However, as suggested in the paper, the framework proposed in this work can be combined with other models focusing on the wireless channel. Also, letting the frame failure probability be a variable allows the exploration of the behaviors of MAC schemes under different channel conditions, as done in the last section of this paper.

The proposed framework was used in this work to evaluate pure-asynchronous approaches, enabled by emerging ultra-low power wake-up receivers, to illustrate how the framework can be used to explore new MAC paradigms. Five MAC schemes were modeled using the proposed framework, two leveraging emerging wake-up receivers (TI-WuR and RI$\mathrm{WuR}$ ), and three traditional protocols (PW-MAC, X-MAC and CSMA/CA used by IEEE 802.15.4 beaconless). In addition to analytical evaluation of the pure-asynchronous approach, experimental measurements were conducted to support our conclusions. Results show the benefits enabled by emerging pure-asynchronous schemes, especially when a transmitterinitiated approach is used, in terms of both power consumption and latency.

\section{REFERENCES}

[1] T. Rault, A. Bouabdallah, and Y. Challal, "Energy Efficiency in Wireless Sensor Networks: A Top-Down Survey," Computer Networks, vol. 67, pp. 104-122, July 2014.

[2] P. Huang, L. Xiao, S. Soltani, M. W. Mutka, and N. Xi, "The Evolution of MAC Protocols in Wireless Sensor Networks: A Survey," IEEE Communications Surveys Tutorials, vol. 15, no. 1, pp. 101-120, January 2013.

[3] C. M. Grinstead and J. L. Snell, Grinstead and Snell's Introduction to Probability. Chance Project, 2006.

[4] Q. Zhao and L. Tong, "A Dynamic Queue Protocol For Multiaccess Wireless Networks With Multipacket Reception," IEEE Transactions on Wireless Communications, vol. 3, no. 6, pp. 2221-2231, November 2004.

[5] Y. Wang, M. Vuran, and S. Goddard, "Cross-Layer Analysis of the Endto-End Delay Distribution in Wireless Sensor Networks," IEEE/ACM Transactions on Networking, vol. 20, no. 1, pp. 305-318, February 2012.

[6] L. Tang, Y. Sun, O. Gurewitz, and D. B. Johnson, "PW-MAC: An Energy-Efficient Predictive-Wakeup MAC Protocol for Wireless Sensor Networks," in IEEE INFOCOM, April 2011, pp. 1305-1313.

[7] Y. Sun, O. Gurewitz, and D. B. Johnson, "RI-MAC: A Receiver-initiated Asynchronous Duty Cycle MAC Protocol for Dynamic Traffic Loads in Wireless Sensor Networks," in ACM Conference on Embedded Network Sensor Systems (SenSys), New York, NY, USA, November 2008, pp. $1-14$. 
[8] N. S. Mazloum and O. Edfors, "Performance Analysis and Energy Optimization of Wake-Up Receiver Schemes for Wireless Low-Power Applications," IEEE Transactions on Wireless Communications, vol. 13, no. 12, pp. 7050-7061, December 2014.

[9] J. Oller, I. Demirkol, J. Casademont, J. Paradells, G. U. Gamm, and L. Reindl, "Has Time Come to Switch From Duty-Cycled MAC Protocols to Wake-Up Radio for Wireless Sensor Networks?" IEEE/ACM Transactions on Networking, vol. 24, no. 2, pp. 674-687, April 2016.

[10] F. Sutton, B. Buchli, J. Beutel, and L. Thiele, "Zippy: On-Demand Network Flooding," in ACM Conference on Embedded Networked Sensor Systems (SenSys), November 2015, pp. 45-58.

[11] D. Spenza, M. Magno, S. Basagni, L. Benini, M. Paoli, and C. Petrioli, "Beyond Duty Cycling: Wake-up Radio with Selective Awakenings for Long-Lived Wireless Sensing Systems," in IEEE INFOCOM, April 2015, pp. 522-530.

[12] H. Yomo, K. Abe, Y. Ezure, T. Ito, A. Hasegawa, and T. Ikenaga, "RadioOn-Demand Sensor and Actuator Networks (ROD-SAN): System Design and Field Trial," in IEEE Global Communications Conference (GLOBECOM), December 2015, pp. 1-6.

[13] V. R. K. Ramachandran, B. J. van der Zwaag, N. Meratnia, and P. J. Havinga, "Evaluation of MAC Protocols with Wake-up Radio for Implantable Body Sensor Networks," in International Conference on Selected Topics in Mobile \& Wireless Networking (MoWNet), September 2014 , pp. $173-180$.

[14] M. Buettner, G. V. Yee, E. Anderson, and R. Han, "X-MAC: A Short Preamble MAC Protocol for Duty-cycled Wireless Sensor Networks," in Proceedings of the 4th International Conference on Embedded Networked Sensor Systems (SenSys), October 2006.

[15] B. Bougard, F. Catthoor, D. C. Daly, A. Chandrakasan, and W. Dehaene, "Energy Efficiency of the IEEE 802.15.4 Standard in Dense Wireless Microsensor Networks: Modeling and Improvement Perspectives," in Conference on Design, Automation and Test in Europe (DATE), March 2005, pp. 196-201 Vol. 1.

[16] I. Ramachandran, A. K. Das, and S. Roy, "Analysis of the Contention Access Period of IEEE 802.15.4 MAC," ACM Transaction on Sensor Networks (ToSN), vol. 3, no. 1, March 2007.

[17] S. Pollin, M. Ergen, S. C. Ergen, B. Bougard, L. V. D. Perre, I. Moerman, A. Bahai, P. Varaiya, and F. Catthoor, "Performance Analysis of Slotted Carrier Sense IEEE 802.15.4 Medium Access Layer," IEEE Transactions on Wireless Communications, vol. 7, no. 9, pp. 3359-3371, September 2008.

[18] P. Park, P. D. Marco, P. Soldati, C. Fischione, and K. H. Johansson, "A Generalized Markov Chain Model for Effective Analysis of Slotted IEEE 802.15.4," in IEEE International Conference on Mobile Adhoc and Sensor Systems, October 2009, pp. 130-139.

[19] X. Vilajosana, Q. Wang, F. Chraim, T. Watteyne, T. Chang, and K. S. J. Pister, "A Realistic Energy Consumption Model for TSCH Networks," IEEE Sensors Journal, vol. 14, no. 2, pp. 482-489, February 2014.

[20] M. C. Vuran and I. F. Akyildiz, "Spatial correlation-based collaborative medium access control in wireless sensor networks," IEEE/ACM Transactions on Networking, vol. 14, no. 2, pp. 316-329, April 2006.

[21] K. Langendoen and A. Meier, "Analyzing MAC Protocols for Low DataRate Applications," in ACM Transactions on Sensor Networks (ToSN), vol. 7, no. 1, 2010, pp. 1-34.

[22] A. Asudeh, G. V. Záruba, and S. K. Das, "A General Model For MAC Protocol Selection in Wireless Sensor Networks," Ad Hoc Networks, vol. 36, Part 1, pp. 189 - 202, January 2016.

[23] Y. Liu, Y. He, M. Li, J. Wang, K. Liu, and X. Li, "Does Wireless Sensor Network Scale? A Measurement Study on GreenOrbs," IEEE Transactions on Parallel and Distributed Systems, vol. 24, no. 10, pp. 1983-1993, October 2013.

[24] M. Haenggi, J. G. Andrews, F. Baccelli, O. Dousse, and M. Franceschetti, "Stochastic geometry and random graphs for the analysis and design of wireless networks," IEEE Journal on Selected Areas in Communications, vol. 27, no. 7, pp. 1029-1046, September 2009.

[25] O. Berder and O. Sentieys, "PowWow : Power Optimized Hardware/Software Framework for Wireless Motes," in International Conference on Architecture of Computing Systems (ARCS), February 2010, pp. 1-5.

[26] M. Magno, V. Jelicic, B. Srbinovski, V. Bilas, E. Popovici, and L. Benini, "Design, Implementation, and Performance Evaluation of a Flexible Low-Latency Nanowatt Wake-Up Radio Receiver," IEEE Transactions on Industrial Informatics, vol. 12, no. 2, pp. 633-644, April 2016.

[27] Y. Ammar, S. Bdiri, and F. Derbel, "An Ultra-Low Power Wake Up Receiver with Flip Flops Based Address Decoder," in International
Multi-Conference on Systems, Signals and Devices (SSD), March 2015, pp. $1-5$.

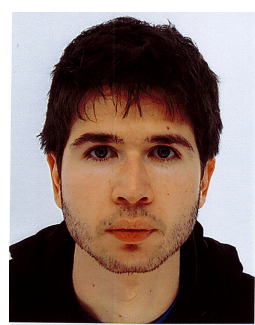

Fayçal Ait Aoudia received his Masters degree in computer science engineering from the National Institute of Applied Sciences of Lyon (INSA Lyon) in 2014. He is currently a Ph.D student at the University of Rennes 1 . He was a visitor Ph.D student at the ETHZ between September 2015 and April 2016.

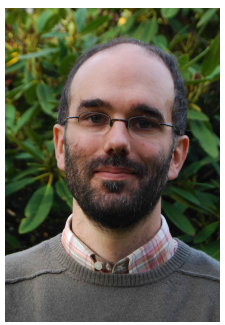

Matthieu Gautier holds the Engineering and M.Sc degrees in electronics and signal processing engineering from the ENSEA graduate school and a $\mathrm{Ph} . \mathrm{D}$. degree from Grenoble-INP in 2006. From 2006 to 2011, he was a research engineer at Orange labs, INRIA and CEA-LETI laboratory. He is currently an associate professor at the University of Rennes and the IRISA Laboratory since 2011. His research activities are in the two complementary fields of embedded systems and digital communications for energy efficient wireless systems.

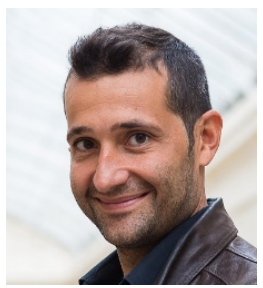

Michele Magno (SM'13) received his Masters and $\mathrm{Ph} . \mathrm{D}$. degrees in electronic engineering from the University of Bologna, Italy, in 2004 and 2010 respectively. Currently he is a Postdoctoral researcher at ETH Zurich, Switzerland and research fellow at the University of Bologna. The most important themes of his research are on wireless sensor networks, wearable devices, energy harvesting, low power management techniques and extension of lifetime of batteries-operating devices. He has collaborated with several universities and research centers, such as University College Cork and Tyndall Institute, Ireland, Imperial College London, UK, University of Trento, Italy, Politecnico di Torino, Italy, University of British Columbia, Canada, among others. He is IEEE senior member and he has published more than 70 papers in international journals and conferences.

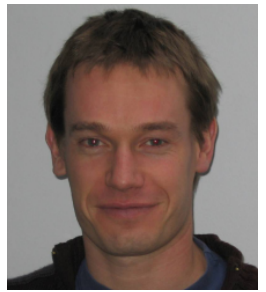

Olivier Berder received the Ph.D. degrees in electrical engineering from the University of Bretagne Occidentale, Brest, in 2002. After a post-doctoral position at Orange Labs, in March 2005 he joined University of Rennes 1 where he is currently a Full Professor at IUT Lannion, and the leader of GRANIT team of IRISA Laboratory. His research interests focus on multiantenna systems and cooperative techniques for energy efficient mobile communications and wireless sensor networks. He has co-authored more than 80 peer reviewed papers in international journals or conferences and has participated in numerous collaborative projects founded by either European Union or French National Research Agency, mainly related to Wireless Sensor Networks. 


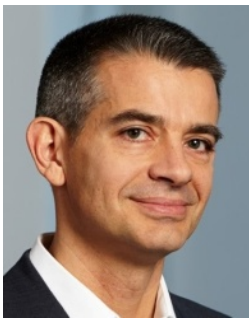

Luca Benini holds the chair of digital Circuits and systems at ETHZ and is Full Professor at the Universita di Bologna. He received a Ph.D. degree in electrical engineering from Stanford University in 1997. Dr. Benini's research interests are in energyefficient system design for embedded and highperformance computing. He is also active in the area of energy-efficient smart sensors and ultra-low power VLSI design. He has published more than 700 papers in peer-reviewed international journals and conferences, four books and several book chapters. He is a Fellow of the IEEE and the ACM and a member of the Academia Europaea. He is the recipient of the 2016 IEEE CAS Van Valkenburg award. 